\title{
Gradient Estimates for the Nonlinear Parabolic Equation with Two Exponents on Riemannian Manifolds
}

\author{
Songbo Hou
}

\begin{abstract}
In this paper, we study the nonlinear parabolic equation with two exponents on complete noncompact Riemannian maniflods. The special types of such equation include the Fisher-KPP equation, the parabolic Allen-Cahn equation and the NewellWhitehead equation. We get the Souplet-Zhang's gradient estimates for the positive solutions to such equation. We also obtain the Liouville theorem for positive ancient solutions. Our results extend those of Souplet-Zhang (Bull. London. Math. Soc. 38 (2006), 1045-1053) and Zhu (Acta Math. Sci. Ser. B 36 (2016), no. 2, 514-526).
\end{abstract}

\section{Introduction}

Let $M$ be a complete noncompact Riemannian manifold. In this paper, we consider the following nonlinear parabolic equation

$$
\frac{\partial u}{\partial t}=\Delta u(x, t)+\lambda(x, t) u^{p}+\eta(x, t) u^{q}
$$

on $M$, where the functions $\lambda$ and $\eta$ are $C^{1}$ in $x$ and $C^{0}$ in $t, p$ and $q$ are positive constants with $p \geq 1, q \geq 1$. If $\lambda=-\eta=c, p=1$ and $q=2$, where $c$ is a positive constant, then the equation (1.1) becomes

$$
\frac{\partial u}{\partial t}=\Delta u+c u(1-u)
$$

which is called the Fisher-KPP equation [6, 11. It describes the propagation of an evolutionarily advantageous gene in a population and has many applications. Cao, Liu, Pendleton and Ward 4 derived some differential Harnack estimates for positive solutions to (1.2) on Riemannian manifolds. Geng and the author [7] extended the result of [4]. If $\lambda=1, \eta=-1, p=1$ and $q=3$, then (1.1) becomes

$$
\frac{\partial u}{\partial t}=\Delta u-\left(u^{3}-u\right)
$$

which is called the parabolic Allen-Cahn equation. A Harnack inequality for this equation was studied in [1]. The gradient estimates for the elliptic Allen-Cahn equation on

Received February 12, 2019; Accepted April 5, 2020.

Communicated by Duy-Minh Nhieu.

2010 Mathematics Subject Classification. 35K55, 58J35.

Key words and phrases. gradient estimate, nonlinear parabolic equation, Liouville theorem. 
Riemannian manifolds were obtained by the author in [9]. The special type of [1.1] also includes the Newell-Whitehead equation 16

$$
\frac{\partial u}{\partial t}=\Delta u+a u-b u^{3}
$$

where $a$ and $b$ are positive constants. It is used to model the change of concentration of a substance. The reader may refer to 2 for the recent results for such equation.

The gradient estimate is an important method in study on parabolic and elliptic equations. It was first proved by Yau [19] and Cheng-Yau [5], and was further developed by Li-Yau [13, Li [12], Hamilton [8], Negrin [15], Souplet and Zhang [17], Ma [14], Yang [18], etc. In [17], Souplelt and Zhang considered the heat equation

$$
\frac{\partial u}{\partial t}=\Delta u
$$

and proved the following result.

Theorem 1.1. Let $M$ be an $n$-dimensional Riemannian manifold with $n \geq 2$ and $\operatorname{Ricci}(M) \geq-k, k \geq 0$. If $u$ is any positive solution to (1.3) in $Q_{R, T} \equiv B\left(x_{0}, R\right) \times\left[t_{0}-\right.$ $\left.T, t_{0}\right] \subset M \times(-\infty,+\infty)$ and $u \leq N$ in $Q_{R, T}$, then there holds

$$
\frac{|\nabla u(x, t)|}{u(x, t)} \leq c\left(\frac{1}{R}+\frac{1}{T^{1 / 2}}+\sqrt{k}\right)\left(1+\log \frac{N}{u(x, t)}\right)
$$

in $Q_{R / 2, T / 2}$, where $c=c(n)$.

Later, using the method of Souplet and Zhang, Zhu [20] studied the equation

$$
\left(\Delta-\frac{\partial}{\partial t}\right) u(x, t)+h(x, t) u^{p}(x, t)=0, \quad p>1
$$

on compete noncomapct Riemannian manfolds, where the function $h(x, t)$ is assumed to be $C^{1}$ in the first variable and $C^{0}$ in the second variable. He proved the following result.

Theorem 1.2. Let $M$ be an $n$-dimensional Riemannian manifold with $n \geq 2$ and $\operatorname{Ricci}(M) \geq-k, k \geq 0$. If $u$ is any positive solution to 1.4 in $Q_{R, T} \equiv B\left(x_{0}, R\right) \times\left[t_{0}-\right.$ $\left.T, t_{0}\right] \subset M \times(-\infty,+\infty)$ and $u \leq N$ in $Q_{R, T}$, then for any $\beta \in(0,2)$, there exists a constant $c=c(n, p, \beta)$ such that

$$
\frac{|\nabla u(x, t)|^{2}}{u(x, t)^{\beta}} \leq c N^{2-\beta}\left(\frac{1}{R^{2}}+\frac{1}{T}+k+N^{p-1}\left\|h^{+}\right\|_{L^{\infty}\left(Q_{R, T}\right)}+N^{\frac{2}{3}(p-1)}\|\nabla h\|_{L^{\infty}\left(Q_{R, T}\right)}^{2 / 3}\right)
$$

in $Q_{R / 2, T / 2}$, where $h^{+}=\max \{h, 0\}$. 
The same method was also used by Huang and Ma [10] to obtain gradient estimates for the equations

$$
\frac{\partial u}{\partial t}=\Delta u+\lambda u^{\alpha} \quad \text { and } \quad \frac{\partial u}{\partial t}=\Delta u+a u \log u+b u
$$

under the Ricci flow, where $\lambda, \alpha, a$ and $b$ are constants.

In this paper, we get the following result.

Theorem 1.3. Let $M$ be an $n$-dimensional Riemannian manifold with $n \geq 2$ and $\operatorname{Ricci}(M) \geq-k, k \geq 0$. Suppose that $\lambda(x, t)$ and $\eta(x, t)$ are $C^{1}$ in $x$ and $C^{0}$ in $t, p$ and $q$ are positive constants with $p \geq 1, q \geq 1$. If $u$ is any positive solution to (1.1) in $Q_{R, T} \equiv B\left(x_{0}, R\right) \times\left[t_{0}-T, t_{0}\right] \subset M \times(-\infty,+\infty)$ and $u \leq N$ in $Q_{R, T}$, then there exists a constant $c=c(n, p, q)$ such that

$$
\begin{aligned}
\frac{|\nabla u(x, t)|}{u(x, t)} \leq c & \left(\frac{1}{R}+\frac{1}{\sqrt{T}}+\sqrt{k}+N^{(p-1) / 2}\left\|\lambda^{+}\right\|_{L^{\infty}\left(Q_{R, T}\right)}^{1 / 2}+N^{(q-1) / 2}\left\|\eta^{+}\right\|_{L^{\infty}\left(Q_{R, T}\right)}^{1 / 2}\right. \\
& \left.+N^{\frac{1}{3}(p-1)}\|\nabla \lambda\|_{L^{\infty}\left(Q_{R, T}\right)}^{1 / 3}+N^{\frac{1}{3}(q-1)}\|\nabla \eta\|_{L^{\infty}\left(Q_{R, T}\right)}^{1 / 3}\right)\left(1+\log \frac{N}{u}\right)
\end{aligned}
$$

in $Q_{R / 2, T / 2}$, where $\lambda^{+}=\max \{\lambda, 0\}, \eta^{+}=\max \{\eta, 0\}$.

Note that the estimate 1.5 is equivalent to

$$
\begin{aligned}
& \frac{|\nabla u(x, t)|}{u(x, t)} \\
\leq & c\left(\frac{N}{u}\right)^{1-\beta / 2}\left(\frac{1}{R}+\frac{1}{\sqrt{T}}+\sqrt{k}+N^{(p-1) / 2}\left\|h^{+}\right\|_{L^{\infty}\left(Q_{R, T}\right)}^{1 / 2}+N^{\frac{1}{3}(p-1)}\|\nabla h\|_{L^{\infty}\left(Q_{R, T}\right)}^{1 / 3}\right) .
\end{aligned}
$$

Applying Theorem 1.3 to 1.4 yields

$$
\begin{aligned}
& \frac{|\nabla u(x, t)|}{u(x, t)} \\
\leq & c\left(\frac{1}{R}+\frac{1}{\sqrt{T}}+\sqrt{k}+N^{(p-1) / 2}\left\|h^{+}\right\|_{L^{\infty}\left(Q_{R, T}\right)}^{1 / 2}+N^{\frac{1}{3}(p-1)}\|\nabla h\|_{L^{\infty}\left(Q_{R, T}\right)}^{1 / 3}\right)\left(1+\log \frac{N}{u}\right) .
\end{aligned}
$$

Since $\lim _{x \rightarrow+\infty} \frac{\log x}{x^{1-\beta / 2}}=0$, if $N / u$ is large enough, then we have

$$
1+\log \frac{N}{u} \leq\left(\frac{N}{u}\right)^{1-\beta / 2}
$$

So in this sense, the estimate (1.6) improves 1.5 .

We also get the Liouville type theorem.

Theorem 1.4. Let $M$ be an n-dimensional Riemannian manifold with nonnegative Ricci curvature. Suppose that $\lambda, \eta$ are nonpositive constants and one of them is negative, then (1.1) does not admit any positive ancient solution with $u(x, t)=e^{o(d(x)+\sqrt{|t|})}$ near infinity.

The method of the proofs of main theorems comes from $10,17,20$. 


\section{Proofs of main theorems}

\subsection{Proof of Theorem 1.3}

Let $\widetilde{u}=u / N$. Then $\widetilde{u}$ satisfies

$$
\frac{\partial \widetilde{u}}{\partial t}=\Delta \widetilde{u}+\widetilde{\lambda} \widetilde{u}^{p}+\widetilde{\eta} \widetilde{u}^{q}
$$

where $\widetilde{\lambda}=\lambda N^{p-1}, \widetilde{\eta}=\eta N^{q-1}$. Noting $\widetilde{u} \leq 1$, we let

$$
f=\log \widetilde{u}, \quad \omega=|\nabla \ln (1-f)|^{2} .
$$

In view of (2.1), we have

$$
\Delta f+|\nabla f|^{2}+\widetilde{\lambda} e^{(p-1) f}+\widetilde{\eta} e^{(q-1) f}-f_{t}=0 .
$$

By $(2.2)$ and $(2.3)$, we have

$$
\begin{aligned}
\omega_{t}= & \frac{2 f_{i}\left(f_{t}\right)_{i}}{(1-f)^{2}}+\frac{2 f_{j}^{2} f_{t}}{(1-f)^{3}} \\
= & \frac{2 f_{i}\left(f_{j j i}+2 f_{j} f_{j i}+\widetilde{\lambda}_{i} e^{(p-1) f}+\widetilde{\lambda}(p-1) e^{(p-1) f} f_{i}+\widetilde{\eta}_{i} e^{(q-1) f}+\widetilde{\eta}(q-1) e^{(q-1) f} f_{i}\right)}{(1-f)^{2}} \\
& +\frac{2 f_{j}^{2}\left(f_{i i}+f_{i}^{2}+\widetilde{\lambda} e^{(p-1) f}+\widetilde{\eta} e^{(q-1) f}\right)}{(1-f)^{3}} .
\end{aligned}
$$

It follows from the similar calculation that

$$
\begin{aligned}
\Delta \omega & =\frac{2 f_{i j}^{2}+2 f_{j} f_{j i i}}{(1-f)^{2}}+\frac{8 f_{i} f_{i j} f_{j}+2 f_{j}^{2} f_{i i}}{(1-f)^{3}}+\frac{6 f_{i}^{2} f_{j}^{2}}{(1-f)^{4}} \\
& =\frac{2 f_{i j}^{2}+2 f_{j} f_{i i j}+2 R_{i j} f_{i} f_{j}}{(1-f)^{2}}+\frac{8 f_{i} f_{i j} f_{j}+2 f_{j}^{2} f_{i i}}{(1-f)^{3}}+\frac{6 f_{i}^{2} f_{j}^{2}}{(1-f)^{4}}
\end{aligned}
$$

where Bochner's identity is used. Noting that $R_{i j} f_{i} f_{j} \geq-k f_{i}^{2}$, we have

$$
\begin{aligned}
\Delta \omega-\omega_{t} \geq & \frac{2 f_{i j}^{2}-4 f_{i} f_{j} f_{i j}-2 e^{(p-1) f} f_{i} \widetilde{\lambda}_{i}-2 \widetilde{\lambda}(p-1) e^{(p-1) f} f_{i}^{2}}{(1-f)^{2}} \\
& -\frac{2 e^{(q-1) f} f_{i} \widetilde{\eta}_{i}+2 \widetilde{\eta}(q-1) e^{(q-1) f} f_{i}^{2}+2 k f_{i}^{2}}{(1-f)^{2}} \\
& +\frac{8 f_{i} f_{i j} f_{j}-2 f_{j}^{2} f_{i}^{2}-2 \widetilde{\lambda} e^{(p-1) f} f_{j}^{2}-2 \widetilde{\eta} e^{(q-1) f} f_{j}^{2}}{(1-f)^{3}}+\frac{6 f_{i}^{2} f_{j}^{2}}{(1-f)^{4}} .
\end{aligned}
$$

From 2.2, we deduce that

$$
-\frac{2 f}{1-f} \nabla f \nabla \omega=\frac{4 f_{i} f_{i j} f_{j}}{(1-f)^{2}}+\frac{4 f_{i}^{2} f_{j}^{2}-4 f_{i} f_{i j} f_{j}}{(1-f)^{3}}-\frac{4 f_{i}^{2} f_{j}^{2}}{(1-f)^{4}} .
$$


Combining (2.4) and 2.5, we have

$$
\begin{aligned}
\Delta \omega-\omega_{t}-\frac{2 f}{1-f} \nabla f \nabla \omega \geq & \frac{2 f_{i j}^{2}-2 e^{(p-1) f} f_{i} \widetilde{\lambda}_{i}-2 \widetilde{\lambda}(p-1) e^{(p-1) f} f_{i}^{2}}{(1-f)^{2}} \\
& -\frac{2 e^{(q-1) f} f_{i} \widetilde{\eta}_{i}+2 \widetilde{\eta}(q-1) e^{(q-1) f} f_{i}^{2}+2 k f_{i}^{2}}{(1-f)^{2}} \\
& +\frac{4 f_{i} f_{i j} f_{j}+2 f_{j}^{2} f_{i}^{2}-2 \widetilde{\lambda} e^{(p-1) f} f_{j}^{2}-2 \widetilde{\eta} e^{(q-1) f} f_{j}^{2}}{(1-f)^{3}}+\frac{2 f_{i}^{2} f_{j}^{2}}{(1-f)^{4}} .
\end{aligned}
$$

Hölder's inequality implies that

$$
\left|\frac{4 f_{i} f_{i j} f_{j}}{(1-f)^{3}}\right| \leq \frac{2 f_{i j}^{2}}{(1-f)^{2}}+\frac{2 f_{i}^{2} f_{j}^{2}}{(1-f)^{4}} .
$$

Thus we have

$$
\begin{aligned}
& \Delta \omega-\omega_{t}-\frac{2 f}{1-f} \nabla f \nabla \omega \\
\geq & -\frac{2 e^{(p-1) f} f_{i} \widetilde{\lambda}_{i}+2 e^{(q-1) f} f_{i} \widetilde{\eta}_{i}}{(1-f)^{2}}-\frac{2 \widetilde{\lambda}(p-1) e^{(p-1) f} f_{i}^{2}+2 \widetilde{\eta}(q-1) e^{(q-1) f} f_{i}^{2}+2 k f_{i}^{2}}{(1-f)^{2}} \\
& +\frac{2 f_{i}^{2} f_{j}^{2}-2 \widetilde{\lambda} e^{(p-1) f} f_{j}^{2}-2 \widetilde{\eta} e^{(q-1) f} f_{j}^{2}}{(1-f)^{3}} \\
= & 2(1-f) \omega^{2}-2 \widetilde{\lambda}\left(p-1+\frac{1}{1-f}\right) e^{(p-1) f} \omega-2 \widetilde{\eta}\left(q-1+\frac{1}{1-f}\right) e^{(q-1) f} \omega \\
& -\frac{2 e^{(p-1) f} f_{i} \widetilde{\lambda}_{i}+2 e^{(q-1) f} f_{i} \widetilde{\eta}_{i}}{(1-f)^{2}}-2 k \omega .
\end{aligned}
$$

Now we choose a smooth cut-off function $\psi=\psi(x, t)$ with compact support in $Q_{R, T}$ such that

(1) $\psi=\psi(r, t), 0 \leq \psi \leq 1$ with $\psi=1$ in $Q_{R / 2, T / 2}$, where $r=d\left(x, x_{0}\right)$;

(2) $\psi$ is decreasing with respect to $r$;

(3) for any $0<\alpha<1,\left|\partial_{r} \psi\right| / \psi^{\alpha} \leq C_{\alpha} / R,\left|\partial_{r}^{2} \psi\right| / \psi^{\alpha} \leq C_{\alpha} / R^{2}$;

(4) $\left|\partial_{t} \psi\right| / \psi^{1 / 2} \leq C / T$.

Using (2.6), we get

$$
\begin{aligned}
& \Delta(\psi \omega)-2 \frac{\nabla \psi}{\psi} \cdot \nabla(\psi \omega)-(\psi \omega)_{t} \\
\geq & 2(1-f) \psi \omega^{2}-2 \widetilde{\lambda}\left(p-1+\frac{1}{1-f}\right) e^{(p-1) f} \psi \omega-2 \widetilde{\eta}\left(q-1+\frac{1}{1-f}\right) e^{(q-1) f} \psi \omega \\
& -2 k \psi \omega-\frac{2 e^{(p-1) f} f_{i} \widetilde{\lambda}_{i}+2 e^{(q-1) f} f_{i} \widetilde{\eta}_{i}}{(1-f)^{2}} \psi+\frac{2 f}{1-f} \nabla f \nabla(\psi \omega)-\frac{2 f \omega}{1-f} \nabla f \nabla \psi
\end{aligned}
$$




$$
-\frac{2|\nabla \psi|^{2}}{\psi} \omega+(\Delta \psi) \omega-\psi_{t} \omega .
$$

Suppose that $\psi \omega$ attains the maximum at $\left(x_{1}, t_{1}\right)$. The argument in [3] implies that we can assume $x_{1}$ is not in the cut-locus of $M$. Then we have $\Delta(\psi \omega) \leq 0,(\psi \omega)_{t} \geq 0$ and $\nabla(\psi \omega)=0$ at $\left(x_{1}, t_{1}\right)$. It follows that

$$
\begin{aligned}
2(1-f) \psi \omega^{2} \leq & 2 \widetilde{\lambda}\left(p-1+\frac{1}{1-f}\right) e^{(p-1) f} \psi \omega+2 \widetilde{\eta}\left(q-1+\frac{1}{1-f}\right) e^{(q-1) f} \psi \omega \\
& +2 k \psi \omega+\frac{2 e^{(p-1) f} f_{i} \widetilde{\lambda}_{i}+2 e^{(q-1) f} f_{i} \widetilde{\eta}_{i}}{(1-f)^{2}} \psi \\
& +\frac{2 f \omega}{1-f} \nabla f \nabla \psi+\frac{2|\nabla \psi|^{2}}{\psi} \omega-(\Delta \psi) \omega+\psi_{t} \omega .
\end{aligned}
$$

In view of $p \geq 1, q \geq 1$ and $f \leq 0$, we have

$$
\begin{aligned}
& 2 \widetilde{\lambda}\left(p-1+\frac{1}{1-f}\right) e^{(p-1) f} \psi \omega+2 \widetilde{\eta}\left(q-1+\frac{1}{1-f}\right) e^{(q-1) f} \psi \omega \\
\leq & 2 \widetilde{\lambda}^{+} p \psi \omega+2 \widetilde{\eta}^{+} q \psi \omega \\
\leq & \frac{1}{16} \psi \omega^{2}+16 \psi\left(\widetilde{\lambda}^{+} p\right)^{2}+\frac{1}{16} \psi \omega^{2}+16 \psi\left(\widetilde{\eta}^{+} q\right)^{2} \\
\leq & \frac{1}{8} \psi \omega^{2}+16\left(\widetilde{\lambda}^{+} p\right)^{2}+16\left(\widetilde{\eta}^{+} q\right)^{2}
\end{aligned}
$$

where $\widetilde{\lambda}^{+}=\max \{\widetilde{\lambda}, 0\}, \widetilde{\eta}^{+}=\max \{\widetilde{\eta}, 0\}$. Straightforward calculations show

$$
\begin{gathered}
\frac{2 e^{(p-1) f} f_{i} \widetilde{\lambda}_{i}+2 e^{(q-1) f} f_{i} \widetilde{\eta}_{i}}{(1-f)^{2}} \psi \\
\leq \frac{f_{i}^{4}}{2(1-f)^{4}} \psi+\frac{3|\nabla \widetilde{\lambda}|^{4 / 3}}{2(1-f)^{4 / 3}} \psi+\frac{f_{i}^{4}}{2(1-f)^{4}} \psi+\frac{3|\nabla \widetilde{\eta}|^{4 / 3}}{2(1-f)^{4 / 3}} \psi \\
\leq \frac{f_{i}^{4}}{(1-f)^{4}} \psi+\frac{3}{2}\left(|\nabla \widetilde{\lambda}|^{4 / 3}+|\nabla \widetilde{\eta}|^{4 / 3}\right) \\
\leq(1-f) \psi \omega^{2}+\frac{3}{2}\left(|\nabla \widetilde{\lambda}|^{4 / 3}+|\nabla \widetilde{\eta}|^{4 / 3}\right), \\
\left|\frac{2 f \omega}{1-f} \nabla f \nabla \psi\right| \leq 2 \omega^{3 / 2}|f||\nabla \psi|=2\left[\psi(1-f) \omega^{2}\right]^{3 / 4} \frac{|f||\nabla \psi|}{[\psi(1-f)]^{3 / 4}} \\
\leq \frac{1}{8}(1-f) \psi \omega^{2}+c \frac{(f|\nabla \psi|)^{4}}{[\psi(1-f)]^{3}} \\
\leq \frac{1}{8}(1-f) \psi \omega^{2}+c \frac{f^{4}}{R^{4}(1-f)^{3}}, \\
2 k \psi \omega \leq \frac{1}{8}(1-f) \psi \omega^{2}+c k^{2} .
\end{gathered}
$$


By the estimates of Souplet and Zhang [17], we have

$$
\begin{gathered}
\frac{|\nabla \psi|^{2}}{\psi} \omega \leq \frac{1}{8} \psi \omega^{2}+c \frac{1}{R^{4}} \leq \frac{1}{8}(1-f) \psi \omega^{2}+c \frac{1}{R^{4}} \\
-(\Delta \psi) \omega \leq \frac{1}{8} \psi \omega^{2}+c \frac{1}{R^{4}}+c k \frac{1}{R^{2}} \leq \frac{1}{8}(1-f) \psi \omega^{2}+c \frac{1}{R^{4}}+c k \frac{1}{R^{2}}, \\
\left|\psi_{t}\right| \omega \leq \frac{1}{8} \psi \omega^{2}+c \frac{1}{T^{2}} \leq \frac{1}{8}(1-f) \psi \omega^{2}+c \frac{1}{T^{2}} .
\end{gathered}
$$

Combining (2.7)-(2.14), we obtain

$$
\begin{aligned}
\frac{1}{8}(1-f) \psi \omega^{2} \leq & 16\left(\widetilde{\lambda}^{+} p\right)^{2}+16\left(\widetilde{\eta}^{+} q\right)^{2}+\frac{3}{2}\left(|\nabla \widetilde{\lambda}|^{4 / 3}+|\nabla \widetilde{\eta}|^{4 / 3}\right) \\
& +c \frac{f^{4}}{R^{4}(1-f)^{3}}+c k^{2}+c \frac{1}{R^{4}}+c k \frac{1}{R^{2}}+c \frac{1}{T^{2}} .
\end{aligned}
$$

Hence

$$
\begin{aligned}
\psi \omega^{2}\left(x_{1}, t_{1}\right) \leq & c N^{2 p-2}\left\|\lambda^{+}\right\|_{L^{\infty}\left(Q_{R, T}\right)}^{2}+c N^{2 q-2}\left\|\eta^{+}\right\|_{L^{\infty}\left(Q_{R, T}\right)}^{2}+c N^{\frac{4}{3}(p-1)}\|\nabla \lambda\|_{L^{\infty}\left(Q_{R, T}\right)}^{4 / 3} \\
& +c N^{\frac{4}{3}(q-1)}\|\nabla \eta\|_{L^{\infty}\left(Q_{R, T}\right)}^{4 / 3}+c \frac{f^{4}}{R^{4}(1-f)^{4}}+c k^{2}+c \frac{1}{R^{4}}+c \frac{1}{T^{2}} .
\end{aligned}
$$

By above estimate, there holds for all $(x, t)$ in $Q_{R, T}$,

$$
\begin{aligned}
\psi^{2} \omega^{2}(x, t) \leq & c N^{2 p-2}\left\|\lambda^{+}\right\|_{L^{\infty}\left(Q_{R, T}\right)}^{2}+c N^{2 q-2}\left\|\eta^{+}\right\|_{L^{\infty}\left(Q_{R, T}\right)}^{2}+c N^{\frac{4}{3}(p-1)}\|\nabla \lambda\|_{L^{\infty}\left(Q_{R, T}\right)}^{4 / 3} \\
& +c N^{\frac{4}{3}(q-1)}\|\nabla \eta\|_{L^{\infty}\left(Q_{R, T}\right)}^{4 / 3}+c \frac{1}{R^{4}}+c \frac{1}{T^{2}}+c k^{2} .
\end{aligned}
$$

Noting that $\psi(x, t)=1$ in $Q_{R / 2, T / 2}$, we get

$$
\begin{aligned}
\frac{|\nabla f(x, t)|}{1-f(x, t)} \leq & \frac{c}{R}+\frac{c}{\sqrt{T}}+c \sqrt{k}+c N^{(p-1) / 2}\left\|\lambda^{+}\right\|_{L^{\infty}\left(Q_{R, T}\right)}^{1 / 2}+c N^{(q-1) / 2}\left\|\eta^{+}\right\|_{L^{\infty}\left(Q_{R, T}\right)}^{1 / 2} \\
& +c N^{\frac{1}{3}(p-1)}\|\nabla \lambda\|_{L^{\infty}\left(Q_{R, T}\right)}^{1 / 3}+c N^{\frac{1}{3}(q-1)}\|\nabla \eta\|_{L^{\infty}\left(Q_{R, T}\right)}^{1 / 3}
\end{aligned}
$$

Finally we have

$$
\begin{aligned}
\frac{|\nabla u(x, t)|}{u(x, t)} \leq c & \left(\frac{1}{R}+\frac{1}{\sqrt{T}}+\sqrt{k}+N^{(p-1) / 2}\left\|\lambda^{+}\right\|_{L^{\infty}\left(Q_{R, T}\right)}^{1 / 2}+N^{(q-1) / 2}\left\|\eta^{+}\right\|_{L^{\infty}\left(Q_{R, T}\right)}^{1 / 2}\right. \\
& \left.+N^{\frac{1}{3}(p-1)}\|\nabla \lambda\|_{L^{\infty}\left(Q_{R, T}\right)}^{1 / 3}+N^{\frac{1}{3}(q-1)}\|\nabla \eta\|_{L^{\infty}\left(Q_{R, T}\right)}^{1 / 3}\right)\left(1+\log \frac{N}{u}\right) .
\end{aligned}
$$

\subsection{Proof of Theorem 1.4}

We prove it by contradiction. Suppose that $u$ is a positive solution to (1.1). Noting that $\lambda$ and $\eta$ are nonpositive constants, it follows from Theorem 1.3 that

$$
\frac{|\nabla u(x, t)|}{u(x, t)} \leq c\left(\frac{1}{R}+\frac{1}{\sqrt{T}}\right)\left(1+\log \frac{N}{u}\right) .
$$


By the same argument as in the proof of Theorem 1.2 in [17] and Theorem 1.8 in [20], fixing $\left(x_{0}, t_{0}\right)$ and applying (2.15) to $u$ on $B\left(x_{0}, R\right) \times\left[t_{0}-R^{2}, t_{0}\right]$, we get

$$
\frac{\left|\nabla u\left(x_{0}, t_{0}\right)\right|}{u\left(x_{0}, t_{0}\right)} \leq \frac{C}{R}[1+o(R)]
$$

It follows that $\left|\nabla u\left(x_{0}, t_{0}\right)\right|=0$ by letting $R \rightarrow \infty$. Noting $\left(x_{0}, t_{0}\right)$ is arbitrary, we have $u(x, t)=u(t)$. Then by (1.1), we get $\frac{d u}{d t}=\lambda u^{p}+\eta u^{q}$. Without loss of generality, we assume that $\lambda<0$.

If $p>1$, integrating $\frac{d u}{d t}$ on $[t, 0]$ with $t<0$ implies that

$$
\frac{1}{1-p}\left(u^{1-p}(0)-u^{1-p}(t)\right) \leq-\lambda t
$$

Then

$$
u^{p-1}(t) \leq u^{p-1}(0)+(1-p) \lambda t .
$$

This yields that if $t$ is large enough, $u^{p-1}(t)<0$ which contradicts that $u$ is positive.

If $p=1$, we get for $t<0$

$$
\log u(0)-\log u(t) \leq-\lambda t
$$

Hence $u(t) \geq u(0) e^{\lambda t}$, which contradicts $u(x, t)=e^{o(d(x)+\sqrt{|t|})}$ near infinity. We finish the proof.

\section{References}

[1] M. Băileşteanu, A Harnack inequality for the parabolic Allen-Cahn equation, Ann. Global Anal. Geom. 51 (2017), no. 4, 367-378.

[2] D. Booth, J. Burkart, X. Cao, M. Hallgren, Z. Munro, J. Snyder and T. Stone, A differential Harnack inequality for the Newell-Whitehead equation, arXiv:1712.04024.

[3] E. Calabi, An extension of E. Hopf's maximum principle with an application to Riemannian geometry, Duke Math. J. 25 (1958), 45-56.

[4] X. Cao, B. Liu, I. Pendleton and A. Ward, Differential Harnack estimates for Fisher's equation, Pacific J. Math. 290 (2017), no. 2, 273-300.

[5] S. Y. Cheng and S. T. Yau, Differential equations on Riemannian manifolds and their geometric applications, Comm. Pure Appl. Math. 28 (1975), no. 3, 333-354.

[6] R. A. Fisher, The wave of advance of advantageous genes, Ann. Eugenics 7 (1937), no. $4,355-369$. 
[7] X. Geng and S. Hou, Gradient estimates for the Fisher-KPP equation on Riemannian manifolds, Bound. Value Probl. 2018 (2018), no. 25, 12 pp.

[8] R. S. Hamilton, A matrix Harnack estimate for the heat equation, Comm. Anal. Geom. 1 (1993), no. 1, 113-126.

[9] S. Hou, Gradient estimates for the Allen-Cahn equation on Riemannian manifolds, Proc. Amer. Math. Soc. 147 (2019), no. 2, 619-628.

[10] G. Huang and B. Ma, Hamilton-Souplet-Zhang's gradient estimates for two types of nonlinear parabolic equations under the Ricci flow, J. Funct. Spaces 2016 (2016), Art. ID 2894207, 7 pp.

[11] A. N. Kolmogorov, I. G. Petrovsky and N. S. Piskunov, Ètude de l'équation de la diffusion avec croissance de la quantité de matière et son application à un problème biologique, Bull. Univ. Moscou Sér. Internat. A 1 (1937), 1-25; English transl. in: P. Pelcé, Dynamics of Curved Fronts, 105-130, Perspectives in Physics, Academic Press, 1988.

[12] J. Li, Gradient estimates and Harnack inequalities for nonlinear parabolic and nonlinear elliptic equations on Riemannian manifolds, J. Funct. Anal. 100 (1991), no. 2, $233-256$.

[13] P. Li and S.-T. Yau, On the parabolic kernel of the Schrödinger operator, Acta Math. 156 (1986), no. 3-4, 153-201.

[14] L. Ma, Gradient estimates for a simple elliptic equation on complete non-compact Riemannian manifolds, J. Funct. Anal. 241 (2006), no. 1, 374-382.

[15] E. R. Negrín, Gradient estimates and a Liouville type theorem for the Schrödinger operator, J. Funct. Anal. 127 (1995), no. 1, 198-203.

[16] A. C. Newell and J. A. Whitehead, Finite bandwidth, finite amplitude convection, J. Fluid Mech. 38 (1969), no. 2, 279-303.

[17] P. Souplet and Q. S. Zhang, Sharp gradient estimate and Yau's Liouville theorem for the heat equation on noncompact manifolds, Bull. London Math. Soc. 38 (2006), no. $6,1045-1053$.

[18] Y. Yang, Gradient estimates for a nonlinear parabolic equation on Riemannian manifolds, Proc. Amer. Math. Soc. 136 (2008), no. 11, 4095-4102.

[19] S. T. Yau, Harmonic functions on complete Riemannian manifolds, Comm. Pure Appl. Math. 28 (1975), 201-228. 
[20] X. Zhu, Gradient estimates and Liouville theorems for linear and nonlinear parabolic equations on Riemannian manifolds, Acta Math. Sci. Ser. B (Engl. Ed.) 36 (2016), no. $2,514-526$.

Songbo Hou

Department of Applied Mathematics, College of Science, China Agricultural University, Beijing, 100083, China

E-mail address: housb@cau.edu.cn 